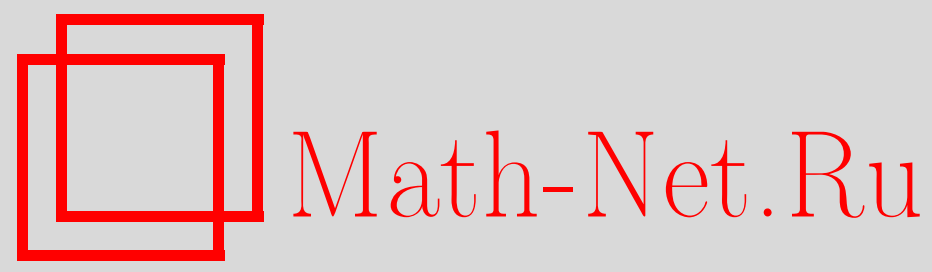

М. Ш. Джамалов, Об асимптотике полиномов Мейкснера, Матем. заметки, 1997, том 62, выпуск 4, 624-625

DOI: https://doi.org/10.4213/mzm1645

Использование Общероссийского математического портала Math-Net.Ru подразумевает, что вы прочитали и согласны с пользовательским соглашением http://www.mathnet.ru/rus/agreement

Параметры загрузки:

IP: 54.174 .149 .18

26 апреля 2023 г., 16:10:45

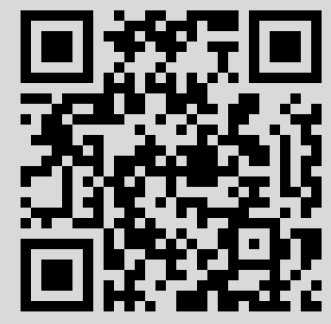




\section{ОБ АСИМПТОТИКЕ ПОЛИНОМОВ МЕЙКСНЕРА}

\section{М.Ш. Джамалов}

Пусть $m_{n}^{\alpha}(x, h), n=0,1,2, \ldots,-$ классические полиномы Мейкснера, ортогональные на сетке $\Omega_{h}=\{0, h, 2 h, \ldots\}$ с весом

$$
p(x)=\left(1-e^{-h}\right)^{\alpha+1} e^{-x} \frac{\Gamma(x / h+\alpha+1)}{\Gamma(x / h+1)},
$$

т.е.

$$
\sum_{x \in \Omega_{h}} m_{n}^{\alpha}(x, h) m_{r}^{\alpha}(x, h) p(x)=\delta_{n r}
$$

где $\alpha>-1, h>0, \Gamma(x)$ - гамма-функция Эйлера, $\delta_{n r}-$ символ Кронекера.

Как известно [1], вопрос об асимптотических свойствах ортогональных полиномов является наиболее трудным в теории этих полиномов. В различных приложениях полиномов $m_{n}^{\alpha}(x, h)$ возникает задача об их асимптотическом поведении при $n \rightarrow \infty, h \rightarrow 0$. В частности, в работе [2] для целых $\alpha>-1$ установлена следующая асимптотическая формула:

$$
m_{n}^{\alpha}(x, h)=\Lambda_{n}^{\alpha}(x)+v_{n}^{\alpha}(x, h)
$$

где

$$
\Lambda_{n}^{\alpha}(x)=\left(\left(\begin{array}{c}
n+\alpha \\
n
\end{array}\right) \Gamma(\alpha+1)\right)^{-1 / 2} L_{n}^{\alpha}(x)
$$

а $L_{n}^{\alpha}(x)$ - классические полиномы Лагерра, в которой при $h \leqslant a / n$ для остаточного члена $v_{n}^{\alpha}(x, h)$ имеет место оценка

$$
\left|v_{n}^{\alpha}(x, h)\right| \leqslant C(a, \alpha)\left(h \sum_{i=0}^{n}\left|\Lambda_{i}^{\alpha}(x)\right|^{2}\right)^{1 / 2} .
$$

Вопрос о справедливости оценки (2) для дробных $\alpha$ оставался открытьм. Основньпм утверждением настоящей заметки является следующая

ТЕОРемА. Пусть $\alpha>-1$ произвольно. Тогда справедлива асимптотическая формула (1), в которой для остаточного члена $v_{n}^{\alpha}(x, h)$ nри $n h \leqslant a, n=1,2, \ldots$, имеет место оценка (2) с константой

$$
C(a, \alpha)=\left(\frac{a^{3} e^{a}}{576}+\left(\frac{5}{6} a+\frac{\alpha+1}{2}\right)^{2} \frac{a}{\alpha+1} \exp \left(a\left(\frac{5}{3} a+\alpha+7\right)\right)\right)^{1 / 2}
$$

Доказательство теоремы опирается на ряд утверждений. Обозначим через $M_{n}^{\alpha}(x, h)$ классические полиномы Мейкснера, ортогональные на сетке $\Omega_{h}$ и нормированные условием

$$
M_{n}(0, h)=\left(\begin{array}{c}
n+\alpha \\
n
\end{array}\right),
$$

и положим $b(h)=\left(e^{h}-1\right) / h, b_{i}(h)=b(i h), i=1,2, \ldots, b_{0}(h)=1$. 
Лемма 1. Пусть $S(r, i), i=0,1,2, \ldots, r, r=0,1,2, \ldots,-$ числа Стирлинга, $\mu_{i n}^{\alpha}(h), i \leqslant n, n=0,1,2, \ldots,-$ коэффициенты разложения

$$
M_{n}^{\alpha}(x, h)=\sum_{i=0}^{n} \mu_{i n}^{\alpha}(h) L_{i}^{\alpha}(x) .
$$

Тогда при $i \leqslant n, n=0,1,2, \ldots$, справедливы соотношения

$$
\mu_{i n}^{\alpha}(h)=b^{n}(h) \sum_{r=i}^{n} \sum_{j=i}^{r}\left(\begin{array}{c}
n+\alpha \\
n-r
\end{array}\right)\left(\begin{array}{c}
j+\alpha \\
j-i
\end{array}\right) \frac{j ! S(r, j)\left(1-e^{h}\right)^{r}}{r ! h^{j}} .
$$

Лемма 2. При $i<n, n=1,2, \ldots$, справедливы соотношения

$$
\begin{gathered}
\mu_{i n}^{\alpha}(h)=b^{n}(h) \sum_{r=i}^{n-1} \int_{0}^{h} b^{-n-1}(t)\left(r b_{n-r+1}^{\prime}(t)-\left(r\left(e^{t}+1\right)+\alpha+1\right) b_{n-r}^{\prime}(t)\right. \\
\left.+(r+\alpha+1) e^{t} b_{n-r-1}^{\prime}(t)\right) \mu_{i r}^{\alpha}(t) d t .
\end{gathered}
$$

Лемма 3. Пусть $\alpha>-1, a>0,0 \leqslant n h \leqslant a, i<n$. Тогда справедливы неравенства

$$
\left|\mu_{i n}^{\alpha}(h)\right| \leqslant C(a, \alpha) h,
$$

әде

$$
C(a, \alpha)=\left(\frac{5}{6} a+\frac{\alpha+1}{2}\right) \exp \left(a\left(\frac{5}{6} a+\frac{\alpha+7}{2}\right)\right) .
$$

Автор выражает благодарность И.И. Шарапудинову за постановку задачи и полезные консультации.

\section{СПИСОК ЦИТИРОВАННОЙ ЛИТЕРАТУРЫ}

1. Сегё Г. Ортогональные многочлены. М.: Физматгиз, 1962. 2. Sharapudinov I. I. // Facta Univ. Ser. Math. Inform. 1991. V. 6. P. 13-21.

Дагестанский государственный педагогический университет, 